\title{
Opportunistic Broadcast of Emergency Messages in Vehicular Ad Hoc Networks with Unreliable Links
}

\author{
(Invited paper) \\ Ming Li and Wenjing Lou \\ Department of Electrical and Computer Engineering \\ Worcester Polytechnic Institute, Worcester, MA 01609, USA \\ \{mingli,wjlou\}@ece.wpi.edu
}

\begin{abstract}
Multi-hop broadcast is an important means to disseminate safety information like time-sensitive emergency messages (EMs) in Vehicular Ad hoc Networks (VANETs). Providing low-latency, highcoverage and scalable multi-hop EM broadcast is a hard task in VANET with unreliable links. The major challenge comes from that fact that the link-layer broadcast uses unreliable transmissions, i.e., no positive feedback to acknowledge the reception of the message. Many existing works have used redundant relay nodes to enhance the reliability of broadcast packet reception. However they often involve more relays than it is necessary, which increases the network load and undermines the scalability of the protocol. Moreover, large latency is often incurred due to coarse protocol design.

In this paper, we propose a new EM broadcast scheme that uses a small number of relays to achieve fast multi-hop EM propagation, at the same time to maintain a high level of transmission reliability, i.e., a minimum packet reception probability (PRP). Two types of relays are introduced to provide fast EM propagation and to enhance PRP simultaneously, so that low-latency, the desired reliability level and small overhead can be achieved at the same time. The opportunistic broadcast protocol (OBP) is based on $o p$ portunistic broadcast (OB), a MAC-layer mechanism to select single relay distributively, which features an effective redundant relay suppressing mechanism and very small rebroadcast delay for high priority nodes. Simulation study shows that OBP achieves close to $100 \%$ PRP, while using a small number of relays with very low broadcast latency under a wide range of road traffic conditions.
\end{abstract}

\section{Categories and Subject Descriptors}

C.2.2 [Computer-Communication Networks]: Network Protocols; C.2.1 [Computer-Communi-cation Networks]: Network Architecture and Design - Wireless Communication

\footnotetext{
*This work was supported in part by the US National Science Foundation under grants CNS-0626601 and CNS-0716306.
}

Permission to make digital or hard copies of all or part of this work for personal or classroom use is granted without fee provided that copies are not made or distributed for profit or commercial advantage and that copies bear this notice and the full citation on the first page. To copy otherwise, to republish, to post on servers or to redistribute to lists, requires prior specific permission and/or a fee.

QShine 2008, July 28-31, 2008, Hong Kong, Hong Kong.

Copyright 2008 ICST ISBN 978-963-9799-26-4

DOI 10.4108/ICST.QSHINE2008.3827

\section{General Terms}

Algorithm, performance

\section{Keywords}

VANET, opportunistic broadcast, reliability, scalability

\section{INTRODUCTION}

Communication in Vehicular Ad Hoc Networks (VANETs) has been an active research area in recent years. Enabled by Dedicated Short Range Communication (DSRC) [1], these networks are designed to provide a wide range of applications such as safety warning, congestion avoidance or mobile infotainment. One of the most important applications of VANET is the multi-hop broadcast of emergency messages (EMs) like hazard warning. Often, EMs need to be sent onto a long backward road segment to notify as many upcoming vehicles as possible and as soon as possible. This necessitates the use of multi-hop broadcast, which extends the broadcast range to several thousands meters. Moreover, the broadcast service needs to have good performance under different traffic scenarios, especially when the network is dense. Therefore, the main goals of EM broadcast are high coverage, low-latency and scalability.

However, in real VANETs these goals are hard to achieve at the same time. The major challenge comes from unreliable wireless links $[19,20]$, which undermine the reliability of single-hop broadcast, the building block of multi-hop broadcast. According to studies on the existing DSRC [18], the one-hop broadcast reception rate is low. This is because channel fading makes the probability of successful packet reception decrease with distance, and packet collisions could rise from hidden terminals due to the lack of channel resource reservation, which gets even worse in a dense network with congested channel. Unlike unicast, there is no positive feedback to acknowledge the reception of a broadcast message. Therefore, no guarantee of packet reception can be made for a single-hop link layer broadcast.

In order to enhance multi-hop broadcast reception rates, most previous works have focused on redundant relay retransmission strategies from network layer. While blind flooding leads to the well-known broadcast storm problem [11] where packet collisions arise due to uncoordinated simultaneous rebroadcasts, various methods were proposed to mitigate this problem, such as probabilitybased methods [21] and temporal ordered retransmissions [12, 16, $17,10,15,3]$. However, they often spend more redundant transmissions than it is necessary which increases channel load and reduces scalability. Moreover, the broadcast latency is often large due to the big time delay needed to separate two subsequent retransmissions to avoid collision. 
Actually, if we define reliability in multi-hop broadcast as the minimum packet reception probability (PRP) among all the nodes, then $100 \%$ reliability cannot be absolutely guaranteed no matter how many relays are used, because the packet reception of onehop broadcast is probabilistic. Also, the reliability enhancement decreases with each additional retransmission. Therefore, we need to strike a balance between reliability and overhead.

In this paper, we propose a new multi-hop EM broadcast scheme for VANET with unreliable links. Our main contributions are twofold. First, the broadcast scheme obtains a nice balance between reliability and overhead, while achieving low latency. The relays are classified into forwarder which provides fast forward-propagation and reduces broadcast latency, and makeup which enhances PRP of nodes. By setting a target minimum PRP, makeups are successively chosen until the specified PRP is reached. The selection process of makeups is heuristically optimized so that the total number of relays is reduced.

Second, we propose a distributed opportunistic broadcast protocol (OBP) to actually select those relays, where the basic component is Opportunistic Broadcast (OB). The OB takes advantage of the unreliable wireless channel, in that the node with the biggest priority among the receiver set of a single broadcast is always chosen as the relay, after it quickly sends a short ACK at base rate to suppress other potential relays. In this way, the number of redundant relays are effectively cut down, and the rebroadcast delay can be very small. NS-2 simulations under highway scenario show that OBP is able to achieve close to $100 \%$ EM coverage with very small latency when the network is well connected; and the scalability to the dense traffic regime in terms of number of relays is good.

\section{PROBLEM STATEMENT}

In this paper, we consider the emergency message broadcast in the highway scenario. Fig. 1 shows the system model, which is a straight highway with two lanes in each direction. The VANET consists of vehicles that are all equipped with On Board Units (OBUs) that can communicate with each other. Suppose an accident happens in the westbound road, where the source vehicle stops and its OBU begins to broadcast Emergency Messages (EMs) toward the Interested Region (IR). The IR is defined as the westbound road segment of length $\mathscr{L}$ in the east of the source vehicle, and the message propagation direction is opposite to the driving direction.

For each EM, the goal of broadcast is to cover the IR with a vehicular packet reception ratio of at least $P_{t h}$. Under this constraint, we want to reduce both the total number of relays and broadcast latency. Next we give several assumptions made in this paper.

- Vehicles are GPS-capable. Each vehicle obtains its location and speed in real-time. This is widely accepted assumption in VANET literature.

- The vehicles have the knowledge of the average traffic density $\rho$ in a sufficient long road segment. This could be a service provided by traffic management.

- Vehicles are aware of the existence and locations of nearby vehicles, as they broadcast beacon messages for every $\delta t=$ $100 \mathrm{~ms}$. This is realistic in the real VANET environment, since for the sake of safety each vehicle must know its distance to others to prevent collision. And it is also required by the WAVE standard [1].

\section{BROADCAST SCHEME}

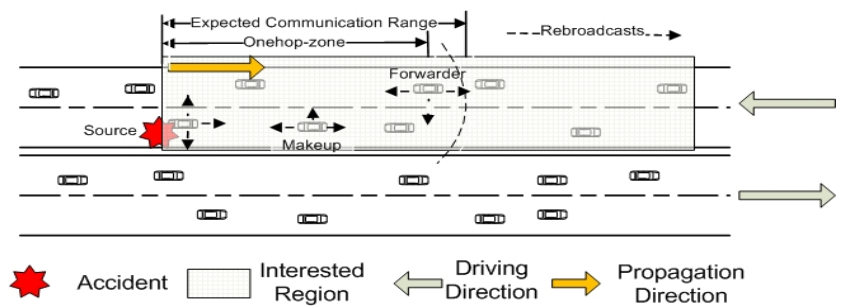

Figure 1: VANET model and overview of the broadcast scheme.

\subsection{Overview}

In this paper, two types of broadcast relays are proposed, i.e., forwarder and makeup. The idea is, first employ one forwarder each hop to greedily advance the EMs in the propagation direction so that the farthermost node can receive EM with small delay; however this results in uncovered nodes between the hops due to probabilistic reception. Therefore makeups are selected to fill the uncovered nodes in the space between two forwarders which is termed as onehop-zone. We aim at reducing the number of forwarders (forwarding hops) thereby the broadcast latency, and also minimizing the number of makeups in each onehop-zone so that a given minimum PRP is reached. The latter provides a balance between reliability and overhead. An overview of the broadcast scheme is shown in Fig. 1. Next we give the definitions.

DEFINITION 1 (FORWARDER). A nodes in the IR that receives a new EM from the source becomes a potential forwarder. The potential forwarder that actually rebroadcasts becomes a forwarder. Similarly, a node that receives a new EM from a forwarder and is in the propagation direction becomes a potential forwarder. The space between two forwarders (or the source and a forwarder) is defined as an onehop-zone.

DEFINITION 2 (MAKEUP). A node located in the onehop-zone and receives a new EM from a forwarder becomes a potential makeup. A potential makeup that actually rebroadcasts become a makeup.

For each type of relay, the broadcast process it is involved in is called forwarding or makeup phase respectively. There is only one forwarding phase, which terminates until the last forwarder completes its job; but there may be multiple makeup phases, each of which consists of makeups in an onehop-zone. The forwarding and makeup phases are done in parallel, because an onehop-zone is defined immediately after another forwarder in the propagation direction broadcasts. Thus, the broadcast latency is reduced. In each broadcast phase, the relays are selected incrementally. The single relay selection is done by $\mathrm{OB}$; and the parameter of $\mathrm{OB}$ is given by the relay selection algorithms.

\subsection{Relay Selection Mechanisms}

\subsubsection{Opportunistic broadcast}

The foundation of our relay selection ${ }^{1}$ mechanisms is $\mathrm{OB}$, which is motivated from opportunistic routing (OR) $[5,22]$ in the multihop wireless network literature. The basic idea of $\mathrm{OB}$ is to calculate a rebroadcast time delay based on the priority of each node that receive a broadcast, and the node with the smallest delay first sends out an ACK at base rate to suppress other nodes before rebroadcast and becomes the actual relay. If the ACK fails to be received due

${ }^{1}$ By "selection" we mean the nodes determine who is the relay after the relay actually rebroadcasts. 


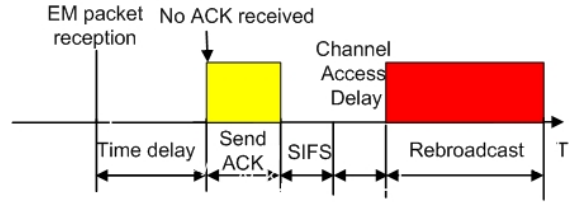

Figure 2: Time domain illustration of $\mathrm{OB}$.

to collision, then another lower-priority node takes the role of the former one. Thus at least one relay is ensured to transmit the EM.

The process of OB is as follows. When a node receives an EM, it first decides whether to set the rebroadcast timer and calculates its time delay. After the timer expires, the relay suppressing is done by a short MAC-layer ACK sent at base rate. After the ACK has been transmitted, the EM is sent from MAC layer. Note that EM still participates in the medium access contention if the channel is busy. The time line of events are shown in Fig. 2.

A key element of $\mathrm{OB}$ is the delay-distance function that takes the following basic form:

$$
\Delta t_{i}=f(d(i, s), R)
$$

where $d(i, s)$ is the distance from the receiving node $i$ to the previous broadcaster $s, R$ is the boundary range so that any node with a distance beyond this range does not participate in contention. The distance represents the node priority in rebroadcast, and the reason will be shown later.

The design of this function is important for the effectiveness of redundant relay suppression. Multiple researchers have adopted the continuous functions, including linear [6, 4, 3] or non-linear ones [9]. The former often cannot distinguish between two nodes near in location, and the latter partially overcomes this by magnifying the delay difference between two nodes near the boundary. However, if there happens to be no node near the boundary the minimum delay of relays will increase greatly. On the other hand, discrete (slotted) delay like LDMA [10] gives enough time separation between nodes in different spatial slots, but they do not distinguish nodes in the same slot. We use an enhanced slotted delay function, where the setting of the unit slot length aims at reducing the number of nodes in one slot, and random delay is used to separate multiple nodes in one slot:

$$
\begin{array}{r}
S\left(x_{i}, R\right)=\left\lfloor\frac{R-d\left(x_{i}, x_{I}\right)}{L}\right\rfloor, L=1000 / \rho, x_{i} \in\left[x_{I}, x_{D}\right] \\
\Delta t_{i}\left(x_{i}, R\right)= \begin{cases}S \cdot(T+\delta)+T \cdot \operatorname{Rand}(0,1), & x_{i} \in\left[x_{I}, x_{D}\right] ; \\
\infty, & \text { otherwise. }\end{cases}
\end{array}
$$

Where $S$ is the slot number, $d\left(x_{i}, x_{j}\right)$ stands for the distance between x-positions of two nodes, $L$ is the spatial slot length ( $\rho$ is the traffic density in \# of vehicles $/ \mathrm{km}$ ), $T$ is the unit time delay for each slot, $\delta$ is the safe interval which is used to separate two neighboring slots, $x_{D}$ is the boundary location towards which the delay should decease; $x_{I}$ is location of previous sender towards which the delay should increase, and $d\left(x_{I}, x_{D}\right)=R$. The delay function is depicted in Fig. 3. In the above, the spatial slot length $L$ is adaptively set to be the average vehicle inter-space, so that on average there will be only one vehicle in each spatial slot. The boundary range $R$ is determined by specific relay selection algorithms.

The OB has several advantages. First, the suppression technique reduces unnecessary relays. The short ACK can be received by more nodes than EM because the base transmission rate requires lower SINR than data rate. Also, since the ACK is very short (the

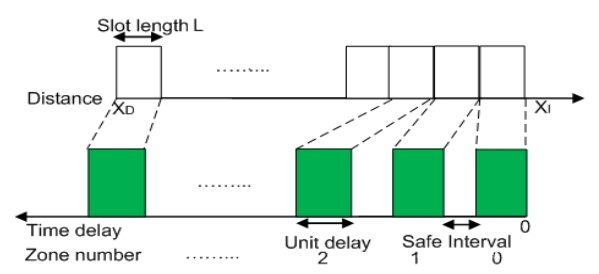

Figure 3: The delay-distance function in $\mathrm{OB}$.

transmission delay of it takes less than $100 \mu \mathrm{s}$ ), the unit delay of one slot can be set small (e.g. 200 $\mu$ s) and the EM reforwarding delay of the chosen relay will be small too. Although the ACK might collide with other packets due to immediate transmission after the delay, the chance of happening is small since ACK is short.

\subsubsection{Forwarder Selection}

The forwarder selection algorithm is to calculate time delay: $\Delta t_{i}\left(x_{i}, R\right)$ according to Eq. 2 for every potential forwarder. Since the broadcast delay is determined mainly by the delay of the forwarding phase, we should minimize the forwarding latency $D_{F}$, which is positively correlated with the number of hops (forwarders): $D_{F}=$ $\sum_{i=1}^{N_{F}} \Delta t_{i}$ and road length $\mathscr{L} \approx \sum_{i=1}^{N_{F}} R_{i}$, where $N_{F}$ is the total number of forwarders, $\Delta t_{i}$ follows Eq. 2 with $x_{D_{i}}=x_{I_{i}}+R$, and $R_{i}$ is the distance from each forwarder to its previous forwarder (onehopzone length). Therefore, to minimize $D_{F}$, both $N_{F}$ and $\Delta t_{i}$ should be minimized.

Now we study the selection of $R$. In the forwarding phase, we name $R$ as Expected Communication Range (ECR). Define the set of nodes that receive an EM from forwarder $F_{i}$ as $\mathscr{N}_{F_{i}}$. In the ideal case, the ECR should be set to the farthest receiving node $(R=$ $\left.\max _{i \in \mathscr{N}_{F_{i}}} R_{i}\right)$ which will then become the forwarder with $\Delta t_{i}=0$ and $N_{F}$ is minimized. However, under probabilistic radio propagation model the farthest receiving node's distance to the sender is randomized which cannot be predicted by the sender. If ECR is too large, $N_{F}$ can be maximized but $\Delta t_{i}$ will grow large; if ECR is too small, $\Delta t_{i}$ could be minimized but $N_{F}$ will increase again. Therefore a balance must be stroked between $\Delta t_{i}$ and $N_{F}$.

We choose the ECR to be the expected distance from the farthest receiving node to the sender, which is a conservative estimation in order to lower the forwarding delay. Here we adopt the Nakagami propagation model [14]. First, denote the one-hop PRP-distance function as $P_{r}(x)$, which is the CCDF of a Gamma distribution according to the model. Then, for any specific topology, the probability that the furthest receiving node $n_{F}$ is $n_{i}$ can be written as (assume node ID increase monotonically in $x$ direction and source $\mathrm{ID}=0)$ :

$$
P\left[n_{F}=n_{i}\right]=P_{r}\left(x_{i}\right) \prod_{j=i+1}^{N}\left(1-P_{r}\left(x_{j}\right)\right)
$$

The ECR can be calculated as the mean of the above distribution:

$$
E C R=\int\left\{\sum_{i=1}^{N} x_{i} \cdot P\left[n_{F}=n_{i}\right]\right\} \cdot f(\mathbf{X}) d \mathbf{X}
$$

where $\mathbf{X}=\left[x_{1}, \ldots, x_{n}\right]$, and $x_{k} \sim \operatorname{Erlang}(k, L)$ if we assume uniform distribution of node positions.

To compute ECR will be too complex in this way. For demonstration of the method, we make some simplifications. First, we use the parameter settings in [18] and set fading parameter $m$ to be 1.0 for $d>150$, and expected receiving power $\Omega \sim x^{-2}$. Therefore $P_{r}(x)$ is reduced to the CCDF of an exponential distribution. Then, 


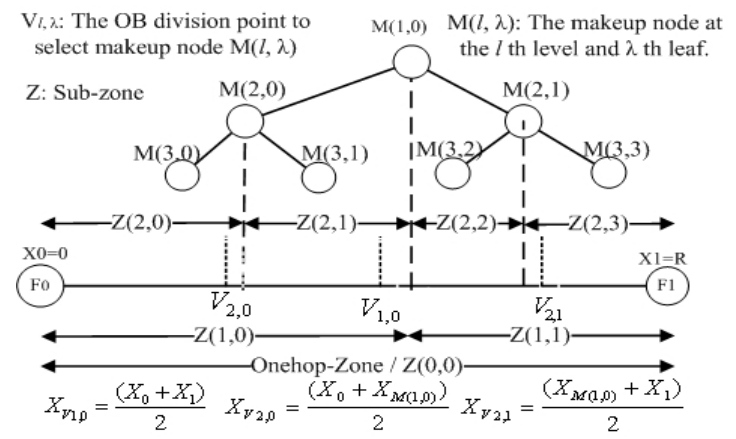

Figure 4: The makeup selection tree, maximum level=3.

because a similar value (hop distance estimation) was calculated in [20] under equal inter-spacing assumption and is shown to be close to the actual value under exponential distribution, we apply their result here:

$$
E C R=\sum_{k=1}^{N} k \cdot s \cdot P_{F}(k, s)
$$

where $s=1000 / \rho, P_{F}(k, s)=P\left[n_{F}=n_{k}\right], x_{k}=k \cdot s$ and $P_{r}\left(x_{i}\right)=$ $\exp \left(-\frac{R_{x t h}}{P_{r e f}} x_{i}^{2}\right) . R_{x t h}$ is the receiving threshold power, and $P_{r e f}$ is the reference power at distance $x_{i}$ calculated from free-space propagation model.

\subsubsection{Makeup Selection}

For each onehop-zone defined by two successive forwarders, we aim at minimizing the number of makeup nodes. A tree based makeup relay selection algorithm is proposed, which is able to achieve the designated minimum PRP with a fairly small number of makeups. The algorithm is heuristic in that each step of makeup selection is optimized. The idea is, the rebroadcast of an upperlevel makeup node divides the original onehop-zone into two subzones, so that new makeup nodes are needed to increase the PRP in each sub-zone. For each sub-zone, we select the makeup that can bring the maximum minimum PRP for all vehicles in that sub-zone. Run this selection process successively and the outcome of makeups form a tree. The makeup selection in each sub-zone terminates whenever the minimum PRP is larger than a predefined threshold $P_{T H}$. Each makeup or sub-zone is numbered by its level and branch $\ell, \lambda$, and the depth of the tree is defined by the maximum levels ${ }^{2}$. An example is shown in Fig. 4.

\section{A. Model-based Estimation of Minimum PRP}

When a potential makeup node $M$ in the sub-zone $\mathbf{Z}_{\ell-\mathbf{1}, \lambda}$ receives an EM from a upper level relay, it first calculates the old minimum PRP among all vehicles belonging to the subset $\mathbf{N}_{L R}=\left\{i \mid x_{L}<x_{i}<\right.$ $\left.x_{R}\right\}$ of nodes in that sub-zone. If the old minimum PRP is already larger than the predefined threshold $P_{T H}$, then it will terminate to broadcast. The expected PRPs for every other node in the same subzone before $M$ 's rebroadcast can be estimated from the propagation model based on locations of all the previous relays on the broadcast path to $M$.

We define the path of locations as $\mathbf{P}_{\mathbf{L}}:\left\{x_{0}, x_{1}, \ldots, x_{n}\right\}$, where the starting points $x_{0}, x_{1}$ are the locations of the left and right forwarders of the current onehop-zone, and $x_{2}, \ldots, x_{n}$ are locations of makeups on the tree branch leading to $M$. For node $M$ satisfying

${ }^{2}$ This will not cause broadcast storm since our basic time delay Eq. 2 is randomized, and the total levels needed is small.
$x_{0}<x_{M}<x_{1}$, the PRP-location function after the rebroadcast of each previous relay in $\mathbf{P}_{\mathbf{L}}$ can be calculated iteratively:

$$
\begin{aligned}
& P_{r_{1}}(x)=1-\left(1-P_{r}\left(x-x_{0}\right)\right)\left(1-P_{r}\left(x_{1}-x\right)\right), x \in\left[x_{L}, x_{R}\right] \\
& P_{r_{n}}(x)=1-\left(1-P_{r}\left(\left|x_{n}-x\right|\right)\right)\left(1-P_{r_{n-1}}(x)\right), x \in\left[x_{L}, x_{R}\right]
\end{aligned}
$$

Note that, we have ignored the contribution from the rebroadcast of relays in other branches of the tree by only counting nodes in $\mathbf{P}_{\mathbf{L}}$, which yields a conservative estimation. At this point, node $M$ calculates the old value of minimum PRP for all the nodes in $\mathbf{N}_{L R}{ }^{3}$ :

$$
\Phi_{\text {min }, \text { old }}\left(\mathbf{N}_{L R}, \mathbf{P}_{\mathbf{L}}\right)=\min _{m \in \mathbf{N}} P_{r_{n}}\left(x_{m}\right)
$$

If $\Phi_{\text {min }, \text { old }}\left(\mathbf{N}_{L R}, \mathbf{P}_{\mathbf{L}}\right)>P_{T H}$, then there is no need for $M$ to rebroadcast. Otherwise, it sets the rebroadcast time delay based on its $P R P$ gain, which is defined as the new value of minimum PRP among all vehicles in the current sub-zone after $M$ rebroadcast.

\section{B. The Calculation of Rebroadcast Time Delay}

In this section, we show that in order to maximize the PRP gain, node's broadcast priority can be mapped into their distance to an optimal point, so that the delay-distance function in OB is directly applicable. Also, we show how to set the boundaries $x_{D}$ and $x_{I}$ for each sub-zone. Here, our goal is to maximize the PRP gain:

$$
\max _{M \in \mathbf{N}} \Phi_{\text {min, new }}\left(\mathbf{N}_{L R}, \mathbf{P}_{\mathbf{L}}, M\right) .
$$

Therefore, the priority of a potential makeup must be positively correlated to the PRP gain after that node rebroadcast.

The new value of minimum PRP of all the nodes in $\mathbf{N}_{L R}$ due to $M$ rebroadcast can be estimated as

$$
\begin{aligned}
& P_{r_{n+1}}(x, M)=1-\left(1-P_{r}\left(\left|x_{M}-x\right|\right)\right)\left(1-P_{r_{n}}(x)\right) \\
& \Phi_{\text {min }, \text { new }}\left(\mathbf{N}_{L R}, \mathbf{P}_{\mathbf{L}}, M\right)=\min _{m \in \mathbf{N}_{\mathbf{L R}}, m \neq M} P_{r_{n+1}}\left(x_{m}, M\right) .
\end{aligned}
$$

Then we want to select the node $M$ that yields the maximum PRP gain. However if the time delay is a continuous function of the difference between $\Phi_{\text {min, new }}$ and $\max _{M \in \mathbf{N}}\left\{\Phi_{\text {min, new }}\right\}$, the indistinguishability problem will arise as analyzed before. Also, the precise value of $\max _{M \in \mathbf{N}}\left\{\Phi_{\text {min, new }}\right\}$ is hard to obtain.

In fact, there is no need to obtain the precise PRP-gain value for each potential makeup, as long as the relative priority among the nodes is the same. The PRP gain-distance mapping is given by the following theorem:

THEOREM 1. Regard the function $P_{r_{i}}(x)$ at iteration $i$ in Eq. 6 as a concave function that is symmetric w.r.t $x_{V}=\frac{x_{L}+x_{R}}{2}$ in $\left[x_{L}, x_{R}\right]$. Then

(1) for an ordered sequence $X_{1}:\left\{x_{V}<x_{i_{0}}<x_{i_{1}}<\ldots<x_{i_{n}}<x_{R}\right\}$ or $X_{2}:\left\{x_{V}>x_{i_{0}}>x_{i_{1}}>\ldots>x_{i_{n}}>x_{L}\right\}$, we have

$$
\min \left\{P_{r_{i+1}}(x, V)\right\}>\min \left\{P_{r_{i+1}}\left(x, i_{0}\right)\right\}>\ldots>\min \left\{P_{r_{i+1}}\left(x, i_{n}\right)\right\} .
$$

(2) A node at location $x_{V}$ results in the maximum PRP gain.

Proof: See Appendix. A.

According to the above theorem, for a sub-zone $\left(\mathbf{Z}_{\ell-\mathbf{1}, \lambda}\right)$ with boundaries $x_{L}$ and $x_{R}$, the optimal boundary $x_{D}$ is the middle point of it $\left(x_{V}\right)$, the priority of nodes in $\mathbf{Z}_{\ell-\mathbf{1}, \lambda}$ decrease with their distance to $x_{V}$. So, for a node $M$ in this sub-zone receiving an EM, its rebroadcast time delay is calculated as $\Delta t_{M}\left(x_{M},\left(x_{R}-x_{L}\right) / 2\right)$ by setting $x_{D}=x_{V}$ (if the old PRP has not yet reached $P_{T H}$ ).

Note that, the above optimality is derived under the assumption that $P_{r_{i}}(x)$ is a symmetric function. In reality, with the level of

${ }^{3}$ For every potential back up in the same onehop-zone, their results of minimum PRP should be the same whenever they share the same neighbor set. 
broadcast increases, $P_{r_{i}}(x)$ deviates from being symmetrical gradually because of the effect of the broadcasts of previous relays. However, the deviation degree is small when the locations of previous relays are far from the current node.

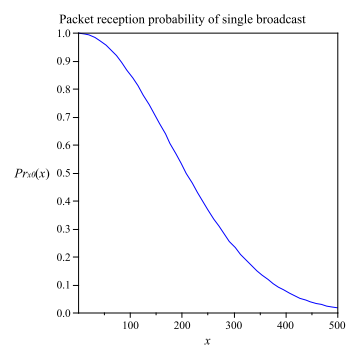

(a)

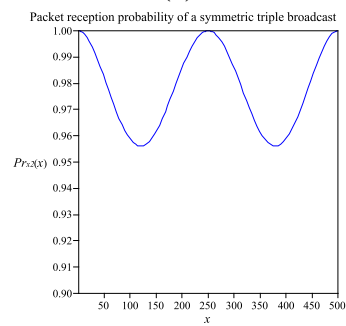

(c)

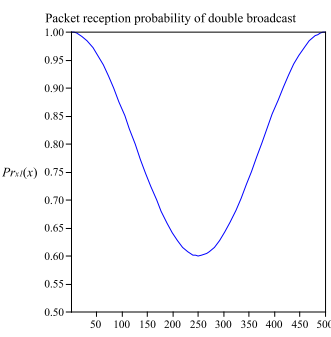

(b)

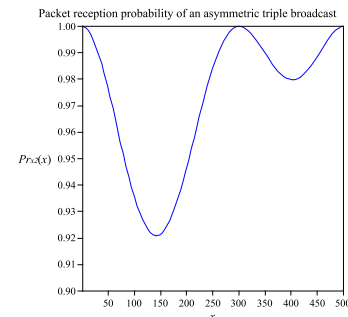

(d)
Figure 5: PRP for consecutive rebroadcasts. (a): Single forwarder broadcast; (b): Double forwarder broadcast (Level 0); (c): Level 1 makeup in the middle of the onehop-zone; (d): Level 1 makeup deviates from the middle point.

Fig. 5 shows an example of how the broadcast reception probability evolves with relays in an onehop-zone. Fig. 5(a) is the onehop PRP $\left(P_{r_{0}}(x)\right)$ of the left forwarder $\left(x_{0}=0\right)$ of an onehop-zone. Fig. 5(b) shows $P_{r_{1}}(x)$ after the right forwarder $x_{1}=500$ rebroadcasts, which is a symmetric function. If there is a node at point $x_{V}=250$, the result is optimal. Though the level 2 PRP function $P_{r_{2}}(x, V), x \in\left[x_{0}, x_{V}\right]$ is not strictly symmetric, the symmetry has not been changed much (Fig. 5(c)), because the impact of the right forwarder decreases fast with distance. Also, even if the level 1 makeup turns out to be at point $x_{i_{k}}=300$, the symmetricalness is almost preserved (Fig. 5(d)).

\section{CROSS-LAYER PROTOCOL DESIGN}

The cross-layer design is used for two considerations. First, EMs should be given the highest priority among all the messages in a VANET thus should be rebroadcasted from MAC layer while being stored in upper layers. Upper-layer broadcast causes additional queuing delays in the interface queues when there is much data traffic going on at a node. Second, to enhance reliability in OB MAC-layer ACK is desired as analyzed in Sec. 3.2.1. We have modified the 802.11 MAC layer reception coordination function to deal with EM. The process is described in Sec. 3.2.1. The MAC is enabled to get EM from upper layer directly when the ACK is sent. Further details are not presented due to space limitation.

In order to enhance the redundant relay suppressing mechanism, an additional rule is used. The ACK from a forwarder in the later onehop-zone suppresses the potential forwarders in the former onehopzones, and a higher level makeup suppresses lower level ones in the same onehop-zone. This is due to the causal relationship between rebroadcasts; when an ACK from a later forwarder is received, we know that forwarders previous to that one must have rebroadcasted.
The same reason applies to makeups, except that causal relationship only exists among the makeups in the same onehop-zone.

\section{PERFORMANCE EVALUATION}

\subsection{Simulation Setup}

We evaluate the performance of OBP, and compare it to CBD [15]. We use the network simulator NS-2.33 [2], which supports the probabilistic propagation model and enhanced 802.11 MAC layers [7]. The parameters are summarized in Table. 1. The other PHY and MAC layer parameters follow the default settings of IEEE 802.11p.

Table 1: Parameter Settings

\begin{tabular}{|l|l|}
\hline Unit delay for a slot & $200 \mu \mathrm{s}$ \\
\hline Safe interval & $100 \mu \mathrm{s}$ \\
\hline ETR for EM and ACK & $250 \mathrm{~m}, 400 \mathrm{~m}$ \\
\hline SINR threshold for EM and ACK & $12 \mathrm{~dB}, 4 \mathrm{~dB}$ \\
\hline Data rates for EM and ACK & $24 \mathrm{Mbps}, 6 \mathrm{Mbps}$ \\
\hline Tx power, CSThresh, Noise floor & $10,-94,-98 \mathrm{dBm}$ \\
\hline EM, Beacon and ACK length & $592,64,14 \mathrm{Bytes}$ \\
\hline Beacon generation rate & 10 packets/s \\
\hline EM generation period & $0.1 \mathrm{~s}$ \\
\hline Vehicle density & $5-100 \mathrm{cars} /(\mathrm{km} \cdot \mathrm{lane})$ \\
\hline Average vehicle speed & $90 \mathrm{~km} / \mathrm{h}$ \\
\hline Road length, IR length & $4 \mathrm{~km}, 2 \mathrm{~km}$ \\
\hline Maximum makeup level & 2 \\
\hline Maximum rebr. delay in CBD & $50 \mathrm{~ms}$ \\
\hline
\end{tabular}

The fading parameter of the Nakagami model is set according to [18]. The data transmission utilizes higher rate for service messages (including EM and beacons) than ACK, and receiving power threshold is set so that service messages and ACKs have different equivalent transmission ranges (ETR) under the Two-Ray-Ground propagation model.

Each vehicle generates beacons at a rate of 10 packets/second for routine safety applications. Event-driven EMs are generated at one vehicle located at $\mathrm{x}=1000 \mathrm{~m}$ in the westbound road every $100 \mathrm{~ms}$ from $2 \mathrm{~s}$ to $3 \mathrm{~s}$. The simulation lasts for $3.5 \mathrm{~s}$.

\subsection{Results}

Fig. 6-Fig. 9 show the mean and the $95 \%$ confidence interval of data points from 10 EMs. A random topology is generated for each road traffic density. First we study the case when $P_{T H}=0.95$.

\subsubsection{Reliability and Scalability}

From Fig. 6 we can see that for all the traffic densities ranging from 20-100 vehicles $/ \mathrm{km} /$ lane, the average packet reception ratio (PRR) of both OBP and CBD are close to 1 with small variations, which means OBP is reliable under a wide range of scenarios. Also, the worst case in the $95 \%$ confidence region is above 0.95 . This is because OBP guarantees a minimum PRP of 0.95 for all the nodes. Moreover, the high PRR is achieved by a small number of relays, which shows good scalability. In Fig. 9 when traffic density grows up, the number of relays used by OBP increases slowly and is much smaller than CBD.

To see the efficacy of relay suppressing mechanisms of OBP, in Fig. 9 we plot the average number of relays per onehop-zone, which includes one forwarder and several makeups. This number increases slowly with traffic density, and is always below 3 . The implication is, only a small number of makeups are needed in each 


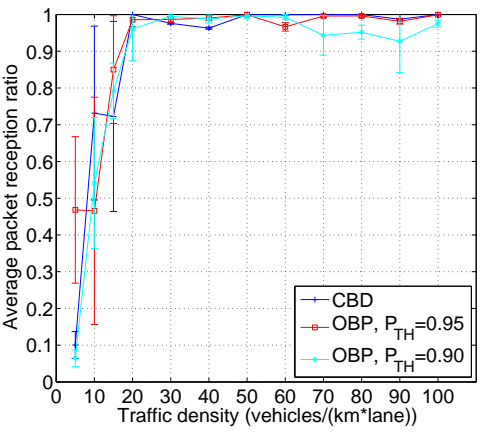

Figure 6: Average packet reception ratio.

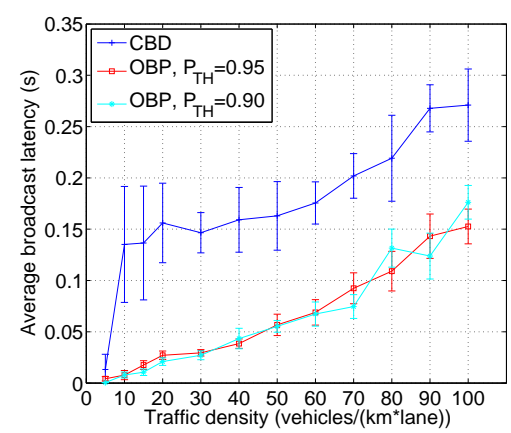

Figure 7: Average broadcast latency

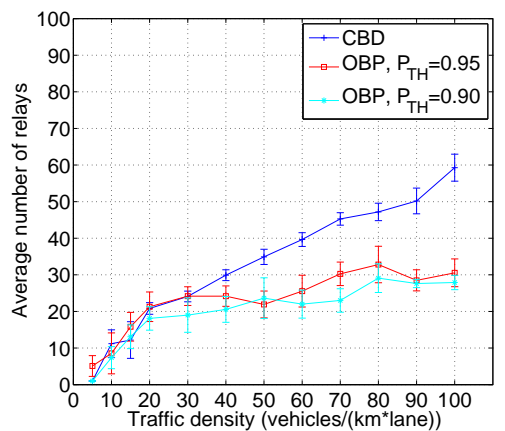

Figure 8: Average number of relays. onehop-zone to ensure larger than 95\% PRP, wherein our case it is less than 2. This is because OBP maximizes the PRP gain for every makeup node, and terminates to select makeups whenever $P_{T H}$ is satisfied. Also, the result reveals the reason that the maximum makeup level being limited to 2 . Although there is indeed some redundant makeups, the redundancy level is low since OBP adopts several mechanisms to suppress unnecessary rebroadcasts.

Note that, the total number of relays first decreases and then increases after some point. Because as traffic density increases, the number of hidden node transmissions grows that makes nodes at larger distance less able to receive an EM, while the ECR increases with traffic density. So the average onehop-zone length first increases and then decreases, which is shown in the inset of Fig. 9. Therefore, the number of onehop-zones (thus relays) first decrease and then increases.

When the traffic density is very small, say $5-10$ vehicles $/ \mathrm{km} / \mathrm{lane}$, both OBP and CBD show bad performance. Due to the long distance between successive vehicles, there is a shortage of relays. The performance enhancement under the sparse traffic regime is out of the scope of this paper.

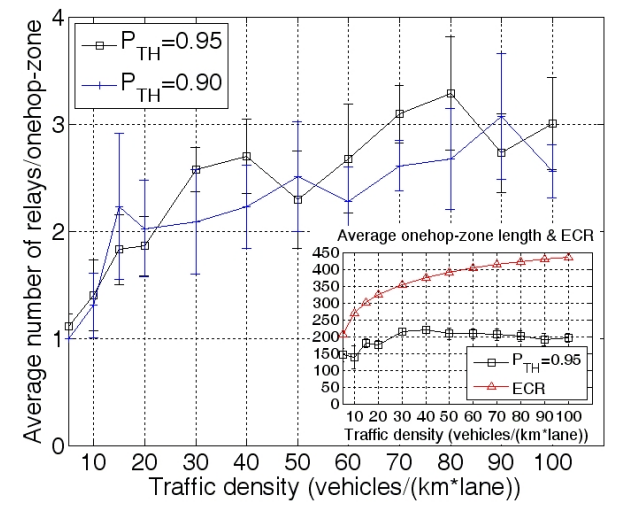

Figure 9: Average number of relays per onehop-zone. Inset: average onehop-zone length and ECR.

\subsubsection{Broadcast Latency}

When the traffic density is moderate (below 80 vehicles $/ \mathrm{km} / \mathrm{lane}$ ), the broadcast latency is below $100 \mathrm{~ms}$, and much smaller than that of CBD (Fig. 7). There are two reasons: first, the total number relays is small so that the last rebroadcast takes fewer time. Second, small one-hop delay is achieved by the cross-layer OBP design. EMs are sent shortly after the ACK is transmitted, while the unit time delay for ACK is set to $200 \mu s$. So even if the slot number of the actual relay is large, its delay will be small. Therefore, with a smaller number of total relays and small one-hop delay, the total latency is made small.

Note that the latency of OBP increases as traffic density grows large. With the increase of density, the number of beacons sent and received by each vehicle in unit time increases which gradually jams the channel. This results in two things. First, it increases the time delay of ACK transmission. The ECR should increase with traffic density, but the actual distance between two forwarders is much smaller than ECR (inset of Fig. 9), which makes $\Delta t$ increase. Second, interference also leads to an inflation of average time delay used for EM packets to access the wireless medium.

On the contrary, the performance of CBD comes at the cost of higher latency, and that more relays are used than OBP and the gap increases with traffic density. First, since CBD utilizes continuous delay function and lengthy EMs to suppress potential relays, enough time gap must be provided between potential relays that are spatially close. This is the reason to set maximum delay to $50 \mathrm{~ms}$, which is a major cause of high latency. Second, the EMs are sent at a high rate which could not suppress as many relays as in OBP using base-rate ACKs. Third, the idea of CBD is to decrease the number of relays from blind flooding by suppressing so that $100 \%$ PRP can be guaranteed. While in OBP the method is to increase the relays from scratch until no more is needed.

\subsubsection{Effect of Changing PRP Threshold}

Now we investigate the performance of OBP under different PRP thresholds. We decrease the value of $P_{T H}$ to 0.90 ; and from the previous figures the average PRR is decreased to larger than $90 \%$. The number of relays is slightly fewer, while the latency is almost the same. Since the increase in PRR is more notable than the decrease in overhead, to set $P_{T H}=0.95$ is comparatively better than $P_{T H}=0.90$ in terms of achieving a better reliability-overhead tradeoff.

\section{RELATED WORK}

The probability based methods [21] use some rebroadcast probability $p$ for each node. They can improve the performance to some extent and need few network information, but the broadcast storm problem could not be avoided since multiple nodes may still send at the same time. To resolve the broadcast storm problem, rebroadcast time delays has been proposed to suppress the retransmission 
of other nodes. The contention based forwarding (CBF) was first proposed to reduce the overhead of unicast [8], and then adopted into VANET and became CBD [20, 15]. To adapt to underlying channel congestion, Oh et.al. [12] combined the timer-based, counter-based and the probability-based methods, where nodes' forwarding probabilities are adjusted according to the inter-arrival time of packets. Also, Alshaer et. al. [3] proposed an optimized adaptive broadcast scheme in which the average rebroadcast probability of each vehicle varies with local node density, and through the setting of random timers the number of relays is minimized. However, the selection of parameters like counter threshold and probability is empirical, and the above schemes result in either surplus relays or over-reduction of retransmissions which decreases packet reception probability in unreliable links. Resta et.al. proposed a similar idea of using two broadcast sub-processes that provide fast propagation and enhance reliability in [13], and the tradeoff between "safety-level" and 1-hop "resource wastage" was analyzed. However, their channel model was oversimplified, and no distributed protocol was proposed.

\section{CONCLUSION}

In this paper, we propose a novel EM broadcast scheme for VANET with unreliable links. We use two types of relay nodes, where the forwarders provide greedy forward propagation, and "a little more than sufficient" number of makeup relays are used to ensure a minimum PRP in each onehop-zone between two forwarders. The relay selection is realized by $\mathrm{OB}$, an efficient distributive mechanism to select single relay, where a relay sends an ACK at base rate to suppress other potential relays before actual EM rebroadcast. The broadcast scheme ensures a desired level of PRP, while the relay selection mechanism together with suppressing rules efficiently reduces the number of relays and enhances scalability. Simulations show that our protocol, OBP, is able to achieve high reliability with small number of relays and low broadcast latency in moderate to dense highway traffic scenarios. We plan to solve the hidden node problem and finding global optimum number of relays in the future works.

\section{REFERENCES}

[1] DSRC and IEEE 1609 standard family, http://www.standards.its.dot.gov/Documents/advisories/ dsrc_advisory.htm.

[2] Ns2.http://www.isi.edu/nsnam/ns.

[3] H. Alshaer and E. Horlait. Optimized adaptive broadcast scheme for inter-vehicle communication. In IEEE VTC, pages 2840-2844, May 2005.

[4] A. Bachir and A. Benslimane. A multicast protocol in ad hoc networks inter-vehicle geocast. VTC'03, 4:2456-2460 vol.4, 22-25 April 2003.

[5] S. Biswas and R. Morris. Exor: opportunistic multi-hop routing for wireless networks. SIGCOMM Comput. Commun. Rev. 35(4):133-144, 2005

[6] L. Briesemeister and G. Hommel. Role-based multicast in highly mobile but sparsely connected ad hoc networks. MobiHoc, pages 45-50, 2000 .

[7] Q. Chen, F. Schmidt-Eisenlohr, D. Jiang, M. Torrent-Moreno, L. Delgrossi, and H. Hartenstein. Overhaul of ieee 802.11 modeling and simulation in ns-2. In MSWiM '07, pages 159-168. ACM, 2007.

[8] H. Füßler, J. Widmer, M. Käsemann, M. Mauve, and H. Hartenstein Contention-based forwarding for mobile ad hoc networks. Ad Hoc Networks, 1(4):351-369, November 2003.

[9] M. Heissenbuttel, T. Braun, M. Walchli, and T. Bernoulli. Optimized stateless broadcasting in wireless multi-hop networks. In INFOCOM, pages 1-12, April 2006.

[10] R. Mangharam, R. Rajkumar, M. Hamilton, P. Mudalige, and F. Bai. Bounded-latency alerts in vehicular networks. In MoVE, pages
55-60, May 2007.

[11] S.-Y. Ni, Y.-C. Tseng, Y.-S. Chen, and J.-P. Sheu. The broadcast storm problem in a mobile ad hoc network. In IEEE/ACM MobiCom, pages 151-162, 1999.

[12] S. Oh, J. Kang, and M. Gruteser. Location-based flooding techniques for vehicular emergency messaging. In IEEE MobiQuitous, pages $1-9$, July 2006

[13] G. Resta, P. Santi, and J. Simon. Analysis of multi-hop emergency message propagation in vehicular ad hoc networks. In $A C M$ MobiHoc, pages 140-149, New York, NY, USA, 2007.

[14] M. K. Simon and M.-S. Alouini. Digital communication over fading channels. Wiley-Interscience, 2005.

[15] M. T-Moreno. Inter-vehicle communications: Assessing information dissemination under safety constraints. In WONS, pages 59-64. IEEE, January 2007.

[16] O. Tonguz, N. Wisitpongphan, F. Bait, P. Mudaliget, and V. Sadekart. Broadcasting in vanet. In MoVE, pages 7-12, May 2007.

[17] O. Tonguz, N. Wisitpongphan, J. Parikh, F. Bai, P. Mudalige, and V. Sadekar. On the broadcast storm problem in ad hoc wireless networks. In BROADNETS, pages 1-11, Oct. 2006.

[18] M. Torrent-Moreno, D. Jiang, and H. Hartenstein. Broadcast reception rates and effects of priority access in 802.11-based vehicular ad-hoc networks. In Proceedings of VANET'04, pages 10-18. ACM, 2004.

[19] M. Torrent-Moreno, M. Killat, and H. Hartenstein. The challenges of robust inter-vehicle communications. IEEE VTC 2005, 1:319-323, 28-25 Sept., 2005.

[20] M. Torrent-Moreno, F. Schmidt-Eisenlohr, H. Fussler, and H. Hartenstein. Effects of a realistic channel model on packet forwarding in vehicular ad hoc networks. IEEE WCNC, 1:385-391, 2006.

[21] N. Wisitpongphan, O. Tonguz, J. Parikh, P. Mudalige, F. Bai, and V. Sadekar. Broadcast storm mitigation techniques in vehicular ad hoc networks. IEEE Wireless Communications, 14(6):84-94, December 2007.

[22] K. Zeng, W. Lou, and H. Zhai. On end-to-end throughput of opportunistic routing in multirate and multihop wireless networks. In IEEE INFOCOM 2008, April 15-17, 2008.

\section{APPENDIX}

\section{A. PROOF OF THEOREM 1}

PROOF. (1): It can be easily seen from the properties of concave and symmetric functions that $x_{V}$ is the minimum point of $P_{r_{i}}(x)$. Then $P_{r_{i+1}}(x, V)$ is also symmetric w.r.t $x_{V}$ :

$$
\begin{aligned}
& P_{r_{i+1}}\left(\left(2 x_{V}-x\right), V\right) \\
& =1-\left(1-P_{r}\left(\left|x_{V}-\left(2 x_{V}-x\right)\right|\right)\right)\left(1-P_{r_{i}}\left(2 x_{V}-x\right)\right) \\
& =1-\left(1-P_{r}\left(\left|x-x_{V}\right|\right)\right)\left(1-P_{r_{i}}(x)\right) \\
& =P_{r_{i+1}}(x, V)
\end{aligned}
$$

So there are two minimal points, $x_{L}^{*}$ and $x_{R}^{*}$ in $x \in\left[x_{L}, x_{V}\right]$ and $x \in$ $\left[x_{V}, x_{R}\right]$ respectively and the two minimal values both equal to the minimum value of $P_{r_{i+1}}(x, V)$ in $\left[x_{L}, x_{R}\right]$. In the following, we pick up the points $x_{i_{0}}$ in sequence $X_{1}$. First, we show that the minimum value of $P_{r_{i+1}}\left(x, i_{0}\right)$ is smaller than that of $P_{r_{i+1}}(x, V)$. At point $x_{L}^{*}$, we have $P_{r_{i+1}}\left(x_{L}^{*}, i_{0}\right)<P_{r_{i+1}}\left(x_{L}^{*}, V\right)$, because

$$
\begin{aligned}
& P_{r_{i+1}}\left(x_{L}^{*}, i_{0}\right)-P_{r_{i+1}}\left(x_{L}^{*}, V\right) \\
& =\left(P_{r}\left(x_{i_{0}}-x_{L}^{*}\right)-P_{r}\left(x_{i_{V}}-x_{L}^{*}\right)\right)\left(1-P_{r_{i}}\left(x_{L}^{*}\right)\right)<0
\end{aligned}
$$

where $P_{r}(x)$ is monotonically decreasing and $x_{i_{0}}>x_{V}$. Therefore, we know that $\min \left\{P_{r_{i+1}}(x, V)\right\}>\min \left\{P_{r_{i+1}}\left(x, i_{0}\right)\right\}$. Immediately we have for any two points $x_{i_{k}}$ and $x_{i_{k+1}}$ in sequence $X_{1}$, we have $\min \left\{P_{r_{i+1}}\left(x, i_{k}\right)\right\}>\min \left\{P_{r_{i+1}}\left(x, i_{k+1}\right)\right\}$. The similar conclusion can be drawn for the sequence $X_{2}$.

(2): This is a direct conclusion from (1). 